\title{
Guest editorial for the special section on MODELS 2014
}

\author{
Juergen Dingel $^{1}$ - Wolfram Schulte ${ }^{2}$
}

Received: 28 August 2016 / Accepted: 1 September 2016 / Published online: 26 September 2016

(C) Springer-Verlag Berlin Heidelberg 2016

\section{Introduction}

The MODELS conference series is the premier venue for model-based software and systems engineering covering all aspects of modeling, from languages and methods to tools and applications. MODELS 2014 took place in Valencia, Spain, from September 28 to October 3, 2014, as the ACM/IEEE 17th International Conference on Model Driven Engineering Languages and Systems. A total of 126 papers were submitted to the 'Foundations' track of MODELS 2014 of which 33 were accepted. The 'MDE in Practice' track received 35 submissions of which 9 were accepted. Thus, both tracks had an acceptance rate of $26 \%$.

It has become a much valued tradition that authors of the best papers at each MODELS conference are invited to submit revised and extended versions of their papers for publication in a special section in SoSyM. The selection of these papers is based on input from the Program Committee and on the reception of the papers at the conference.

This special section presents the six articles that resulted from this invitation. Each article was subject to the full SoSyM review cycle and authors received anonymous feedback in two rounds of reviewing from three reviewers who are experts in the field. As a result, each article has been thoroughly revised and substantially extended compared to its conference version.

\footnotetext{
Juergen Dingel

dingel@cs.queensu.ca

Wolfram Schulte

schulte@microsoft.com

1 Queen's University, Kingston, Canada

2 Microsoft, Redmond, WA, USA
}

\section{Selected papers}

Just like the MODELS 2014 conference program, the selected papers span a large spectrum of topics ranging from embedded and mobile computing, the Internet of Things, model transformations, profiling and annotation, to human factors. The papers also have equally diverse goals: some report on innovative applications of MDE to different software development challenges, while others are more concerned with increasing the capabilities of MDE or contributing to its foundations.

The first three papers belong to the first category and provide interesting, diverse examples of how MDE concepts can be used for the effective development of the many supporting artifacts that large systems and development efforts typically require such as communication glue code, test harnesses, or UML profiles.

The first article, A Model-based approach for the synthesis of software to firmware adapters for use with automatically generated components by Marco Di Natale, David Perillo, Francesco Chirico, Andrea Sindico, and Alberto Sangiovanni-Vincentelli, describes an approach that leverages MDE techniques to facilitate the development of complex electronic defense systems through, e.g., the generation of communication code for subsystems specified with Simulink and of consistent test harnesses to allow testing on the level of both Simulink and $\mathrm{C}++$ code. At the conference, this paper received the 'Best Paper' award in the 'MDE in Practice' track.

The second article is Model-Driven Development of Mobile Applications for Android and iOS Supporting Rolebased App Variability by Steffen Vaupel, Gabriele Taentzer, René Gerlach, and Michael Guckert. It brings the benefits of modeling and code generation to standard aspects of mobile applications development such as implementing data 
management, capturing behavior, and supporting variability. The work nicely demonstrates the efficacy of MDE in new, nonstandard domains such as mobile computing. At the conference, this paper was presented in the 'Foundations' track, just like the all papers discussed below.

The third article Leveraging Annotation-based Modeling with JUMP by Alexander Bergmayr, Michael Grossniklaus, Manuel Wimmer, Gerti Kappel is concerned with facilitating the use of UML and Java for forward and backward engineering tasks. More precisely, the work allows the automatic generation of UML profiles from Java libraries with annotations.

The next three articles are of a more foundational nature and attempt to either characterize the state-of-the-art or advance it by extending the capabilities of MDE or contribute to its foundations.

The fourth article Model-Based Engineering in the Embedded Systems Domain-An Industrial Survey on the State-of-Practice by Grischa Liebel, Nadja Marko, Matthias Tichy, Andrea Leitner, and Jörgen Hansson summarizes the results of a survey among industrial practitioners. The article provides concrete information about what industrial developers think of the use of model-based engineering for the development of embedded systems and which benefits and challenges it entails in their opinion. The work is of interest to anybody interested in the industrial state-of-the-art and how to advance it. At the conference, this paper received the 'Best Paper' award in the 'Foundations' track.

The fifth article On the Impact of Size to the Understanding of UML Diagrams by Harald Störrle focusses on human factors, an often neglected topic. It presents metrics for diagram size, summarizes experiments to measure its impact on diagram understanding, and provides concrete, actionable evidence for the often-used, but still poorly-supported claim that excessive size impedes understanding. The work contributes to an important, but still rather small body of work on how to improve user experience in MDE.

Finally, the sixth article, Foundations for Streaming Model Transformations by Complex Event Processing by István Dávid, István Ráth, and Dániel Varró, considers a new type of model transformation suitable for models found, e.g., in the Internet of Things domain in which models are often too large to be created in their entirety before transformation starts or updated very frequently.

\section{Conclusion}

Just like any other research area, MDE needs research on several, very diverse fronts to thrive and stay relevant, including work that fortifies foundations, determines the state-ofindustrial-practice, presents examples of innovative use, and opens up new areas of application. The MODELS 2014 program very much featured this healthy diversity which is also suitably reflected by the papers in this special section.
Another noteworthy feature of the work presented here is that most of the data that it based on (e.g., questionnaires) and the results that it produced (e.g., source code, survey data) have been made publically available by the authors. While industrial research agreements may not always allow publication of all artifacts, we think it is beneficial to the community because it facilitates related research and increases repeatability.

Acknowledgements We would like to thank the Editors-In-Chief of SoSyM, Bernhard Rumpe, Jeff Gray, and the late Robert France, for their continued support of the long and successful collaboration between MODELS and SoSyM. We also very gratefully acknowledge the work of the referees who spent countless hours producing feedback that greatly improved the submissions. Unfortunately, good reviewing often does not receive the recognition it deserves and needs and this 'thank you', no matter how sincere, does not measure up to the size and importance of our reviewers' efforts. We thank Martin Schindler and Geri Georg from the SoSyM editorial office who have provided valuable assistance with the review process. Last but not least, we thank the authors for submitting their work and thus providing the material that this special issue is made of.

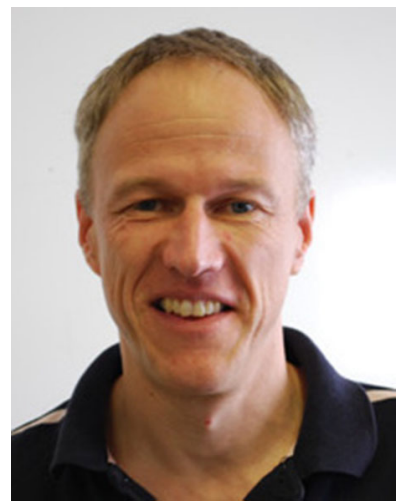

Juergen Dingel received an M.Sc. from Berlin University of Technology in Germany and a Ph.D. in Computer Science from Carnegie Mellon University (2000). He is a Professor in the School of Computing at Queen's University where he leads the Modeling and Analysis in Software Engineering group. His research interests include model-driven engineering, formal methods, and software engineering.

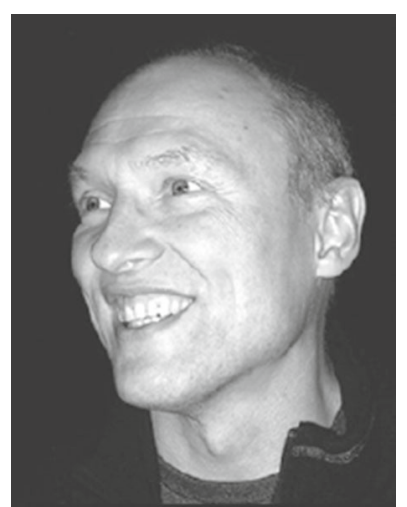

Wolfram Schulte is a Partner Engineering Manager at Microsoft's Cloud and Enterprise Division, Redmond, USA. He currently manages the Tools for Software Engineers (TSE) team, Microsoft's main investment into building a single world-class engineering system, addressing everything from code review, via build, test, and program analysis, to engineering insights. Prior to TSE, he managed the Research in Software Engineering team, one of the world's top software engineering research groups. His research interests include software development tools, ranging from build, via automatic test to deployment, software engineering analytics, ranging from collecting data to prediction, programming languages, and ranging from language design to runtimes. Before joining MSR in 1999, he worked at the University of Ulm (1993-1999), at sd\&m, a German software company (1992-1993), and at the Technical University Berlin (19871992). 